\title{
Comportamiento de la corrosión de aleaciones de magnesio AZ31-B en ambiente marino, modificadas por el proceso de fricción-agitación
}

\author{
Corrosion behavior in marine environment of magnesium alloy AZ31-B \\ welded by friction-agitation process
}

Willian Aperador Chaparro ${ }^{1} \quad$ Geovanny Rodríguez Zamora ${ }^{2} \quad$ Fernando Franco $^{2}$

Recibido 28 de marzo de 2011, aceptado 2 de abril de 2012

Received: March 28, 2011 Accepted: April 2, 2012

\begin{abstract}
RESUMEN
En el presente artículo se estudia el comportamiento de la corrosión de la aleación de magnesio AZ31-B en ambiente marino simulado, modificada mediante el proceso de fricción-agitación (PFA), con el fin de determinar el efecto de las variables del proceso, velocidad de rotación y velocidad de avance. Se llevaron a cabo análisis mediante espectroscopia de impedancia electroquímica y curvas de polarización potencio-dinámicas (Tafel). Adicionalmente, se determinó la microestructura en las zonas del cordón de soldadura a través de metalografía óptica. Finalmente, se analizaron los productos de corrosión formados en la superficie de las muestras por medio del microscopio electrónico de barrido (SEM) equipado con el analizador químico por EDS. Se observó que una relación de velocidad de avance/velocidad de rotación mayor produce menor velocidad de corrosión y con ello mayor resistencia a la corrosión en medios salinos, al parecer relacionados con el gran tamaño de grano en la zona agitada, que corresponde a más entrada de calor.
\end{abstract}

Palabras clave: PFA, aleación de magnesio AZ31, resistencia a la corrosión, velocidad de avance, velocidad de rotación.

\begin{abstract}
The corrosion behavior of AZ31B magnesium alloy modified by friction stir processing (FSP) was studied in simulated marine environment, in order to determine the effect of process variables rotation speed and travel speed. The corrosion analysis was carried upon by means of electrochemical impedance spectroscopy and potentiodynamic polarization curves (Tafel), metallographic analysis of the welded zones was done by optical microscopy and the chemical analysis of the corrosion products were done by using scanning electron microscope (SEM), equipped with EDS analyzer. It was observed that the increase of the rate rotation speed/travel speed of the process produces a decrease in the corrosion rate and the corresponding increase of the corrosion resistance in marine environment, apparently related to the higher grain size found in the stir zone, corresponding to a higher heat input.
\end{abstract}

Keywords: FSP, AZ31 magnesium alloys, corrosion resistance, rotation speed, travel speed,

\footnotetext{
1 Ingeniería Mecatrónica, Universidad Militar Nueva Granada, Carrera 11 No. 101-80 / Conmutador: (57+1) 2757300, Bogotá Colombia. E-mail: g.ing.materiales@gmail.com.

2 Ingeniería de Materiales, Universidad del Valle, Ciudad Universitaria Meléndez Calle 13 № 100-00, Cali Colombia, E-mail: geo-rodri@hotmail.com; fdo_franco@yahoo.com
} 


\section{INTRODUCCIÓN}

La técnica de modificación superficial de aleaciones metálicas mediante el procesamiento por fricciónagitación (PFA) es una alternativa tecnológica de fácil aplicación que permite mejorar superficialmente las propiedades mecánicas y químicas del material, como consecuencia del refinamiento microestructural resultante de la severa deformación plástica y la recristalización dinámica sufrida por el material procesado a alta temperatura. Este proceso está basado en la técnica de soldadura por fricciónagitación [1,2] (FSW, por su sigla en inglés), en la cual se usa una herramienta rotatoria que consiste en un cuerpo cilíndrico con un pin roscado en su extremo para producir el calentamiento por fricción y la subsiguiente mezcla del material en estado sólido. A diferencia del proceso de soldadura, en el que se obtienen uniones soldadas, en esta técnica se trabaja sólo sobre la superficie de una lámina para generar una capa o "recubrimiento" con propiedades mejoradas.

El creciente interés por el uso del magnesio en la industria del transporte aéreo y terrestre, para reducir el consumo de combustible y de emisiones contaminantes, ha tenido como limitación la baja resistencia mecánica y la pobre ductilidad de estas aleaciones en estado de fundición debido a la presencia de porosidad, estructuras dendríticas vastas y fases eutécticas en el material [3,4].

La investigación del PFA aplicado sobre aleaciones base magnesio ha demostrado recientemente que puede conseguirse la homogeneización microestructural efectiva y el refinamiento del grano como resultado de la severa deformación plástica en caliente y la consecuente recristalización dinámica $[5,6]$. La modificación microestructural del material depende de variables del proceso como temperatura, velocidad rotacional, velocidad de avance, profundidad y geometría de la herramienta. Estos parámetros permiten disminuir el tamaño del grano y homogeneizar su distribución. Adicionalmente, afecta propiedades mecánicas como la dureza y la resistencia del material [7].

Gracias a las ventajas metalúrgicas y mecánicas obtenidas, este procesamiento se está utilizando extensamente en diferentes aleaciones a escala industrial. La modificación microestructural mediante PFA ha sido aplicada exitosamente sobre aleaciones fundidas de aluminio A356 y A319, lo que ha producido un significativo incremento en la resistencia mecánica, la ductilidad y la vida a fatiga como resultado de la transformación de la estructura dendrítica mediante el extensivo refinamiento de grano, la fractura y disolución de las partículas de segundas fases gruesas y la eliminación de la porosidad [8].

El problema es que las aleaciones de magnesio, comparadas con materiales como el aluminio o el acero, presentan un potencial electroquímico negativo que las hace más susceptibles de fenómenos de corrosión desde el punto de vista termodinámico. El magnesio revela buena resistencia a la oxidación en ambientes secos a temperatura ambiente. Sin embargo, la vulnerabilidad a la corrosión se incrementa a medida que aumentan la humedad relativa y la temperatura ambiente [9-11].

La razón de la precaria resistencia a la corrosión de aleaciones de magnesio se debe principalmente a dos mecanismos: i) las películas de óxido formadas sobre la superficie no son homogéneas ni protectoras y ii) la corrosión galvánica o bimetálica puede ser causada por la presencia en el material de impurezas y fases secundarias. Últimamente se han realizado estudios sobre el comportamiento de la corrosión de las aleaciones de magnesio. Sin embargo, son escasas en la literatura las investigaciones enfocadas en la corrosión de soldadura por fricción-agitación en las aleaciones de magnesio $[12,13]$.

En este trabajo se evalúa el comportamiento frente a la corrosión marina de las aleaciones de magnesio AZ31-B, modificadas superficialmente mediante el proceso de fricción-agitación con técnicas de evaluación a la corrosión como la espectroscopia de impedancia electroquímica y curvas de polarización Tafel. Además, se evalúan a través de la técnica de microscopia electrónica de barrido las características microestructurales y la composición química de las fases resultantes en los cordones de soldadura antes y después de haberse sometido al proceso de degradación química.

\section{DETALLES EXPERIMENTALES}

Se utilizaron láminas de la aleación de magnesio AZ31-B cortadas de perfiles extruidos en caliente. 
La composición química del material base, obtenida por análisis de fluorescencia de rayos X, se presenta en la Tabla 1.

Tabla 1. Composición química de la aleación de magnesio AZ31-B.

\begin{tabular}{|c|c|}
\hline Elemento & \% en peso \\
\hline $\mathrm{Al}$ & 2,5 a 3.5 \\
\hline $\mathrm{Mn}$ & 0,20 min \\
\hline $\mathrm{Zn}$ & 0,60 a 1,4 \\
\hline $\mathrm{Ca}$ & 0,04 máx. \\
\hline $\mathrm{Si}$ & 0,10 máx. \\
\hline $\mathrm{Cu}$ & 0,05 máx. \\
\hline $\mathrm{Ni}$ & 0,005 máx. \\
\hline $\mathrm{Fe}$ & 0,005 máx. \\
\hline Otros & 0,30 máx. \\
\hline
\end{tabular}

Las placas fueron cortadas a una longitud de $160 \mathrm{~mm}$ por $80 \mathrm{~mm}$ de ancho para la ejecución de los cordones de soldadura. El proceso PFA se realizó utilizando una fresadora universal, acondicionada para el efecto, como se muestra en la Figura 1. Se realizaron las probetas en diferentes condiciones de velocidad de rotación y velocidad de soldadura, manteniendo constantes la penetración y la inclinación de la herramienta, como lo muestra la Tabla 2.

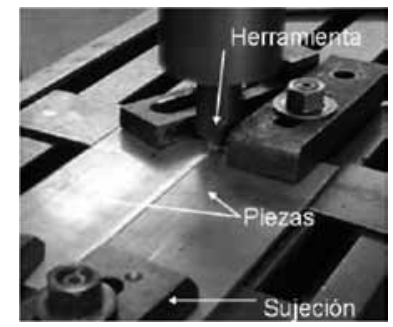

Figura 1. Esquema del montaje utilizado en el proceso de soldadura PFA.

Tabla 2. Variables del proceso PFA.

\begin{tabular}{|l|c|c|c|c|}
\hline \multicolumn{1}{|c|}{ Variable } & \multicolumn{4}{c|}{ Valores } \\
\hline $\begin{array}{l}\text { Vr, Velocidad de } \\
\text { rotación, (rpm) }\end{array}$ & 1500 & 1500 & 750 & 750 \\
\hline $\begin{array}{l}\text { Vs, Velocidad de } \\
\text { soldadura (mm/ } \\
\text { min) }\end{array}$ & 210 & 136 & 105 & 68 \\
\hline $\begin{array}{l}\text { Relación de Vr/ } \\
\text { Vs (rev/mm) }\end{array}$ & 7 & 11 & 7 & 11 \\
\hline $\begin{array}{l}\text { Inclinación de la herramienta, } 1^{\circ} \\
\text { Penetración de la herramienta, } 3 \mathrm{~mm}\end{array}$ \\
\hline
\end{tabular}

Para las pruebas electroquímicas, después de soldar las placas de la aleación se cortaron de forma transversal al cordón de soldadura con un ancho de $15 \mathrm{~mm}$. Después fueron pulidas con diferentes papeles de lija, desde el número 180 hasta el 1000.

Todos los ensayos electroquímicos se realizaron con inmersión de las muestras en una solución con $3,5 \%$ en peso de cloruro de sodio analítico (Panreac PA131655.1211 NaCl-99,0\%). El tipo de electrodo de referencia usado en todos los ensayos fue un electrodo de $\mathrm{Ag} / \mathrm{AgCl}$ de tipo analítico Electrochemistry BAS (MF-2052 RE-5B), el cual tiene un potencial de $+0,194 \mathrm{~V}$ con respecto al electrodo de referencia de hidrógeno a $25^{\circ} \mathrm{C}$. Todos los potenciales están referidos al potencial del electrodo de $\mathrm{Ag} / \mathrm{AgCl}$. En ensayos potenciostáticos y de espectroscopia de impedancias se usó un contraelectrodo de platino. La temperatura de trabajo de los ensayos fue de $25^{\circ} \mathrm{C}$, controlados con una oscilación de $\pm 0,1^{\circ} \mathrm{C}$. Para evitar la contaminación del medio de trabajo, tanto las muestras como los electrodos empleados se limpiaban con agua bidestilada y se secaban al aire antes de sumergirlos en el fluido.

Las técnicas electroquímicas se realizaron según los estándares ASTM G1 y G3 $[14,15]$ relativos a los ensayos de electroquímica.

En la caracterización morfológica se usó un microscopio electrónico de barrido Philips XL 30 FEG de alta resolución, equipado con sistema de EDAX EDX cuya resolución es de 1-nm a $30 \mathrm{kV}$.

\section{RESULTADOS Y DISCUSIÓN}

\section{Análisis de EIS y curvas de polarización Tafel}

En la Figura 2 se muestran los diagramas de Nyquist, correspondientes al material base (al que no se aplicó el proceso de soldadura PFA) y las soldaduras obtenidas; para la aleación de magnesio AZ31-B se encontró la mayor impedancia total, por lo tanto se presentó una velocidad de corrosión más baja. En las soldaduras que se trabajaron con relaciones de velocidad de rotación/velocidad de avance $(\mathrm{Vr} / \mathrm{Va})$ 750/105 y 1500/210 se generaron los menores valores de impedancia total, con respecto a las trabajadas a $750 / 68$ y 1500/136. Esto se debe a que durante el proceso PFA ocurre en la zona central una recristalización dinámica que produce granos finos y equiaxiales por la influencia de los parámetros del 
proceso en la microestructura de las aleaciones de magnesio soldadas con PFA. Se ha observado que el tamaño de los granos recristalizados puede disminuir si decrece la velocidad de rotación para una velocidad de avance constante, o si disminuye la relación de velocidades $\mathrm{Vrel}=\mathrm{Vr} / \mathrm{Va}[1,4]$. El espectro de la aleación de magnesio y las soldaduras consta de un arco capacitivo en el rango de alta frecuencia y un arco inductivo en el de baja frecuencia. El primero puede ser relacionado por la combinación paralela de la capacitancia de la interfaz y la resistencia R1 de transferencia de carga (Figura 2). En este caso, la R1 es usada para evaluar la resistencia a la corrosión de las muestras. El segundo se puede atribuir a películas parcialmente protectoras sobre la superficie de las aleaciones de magnesio.

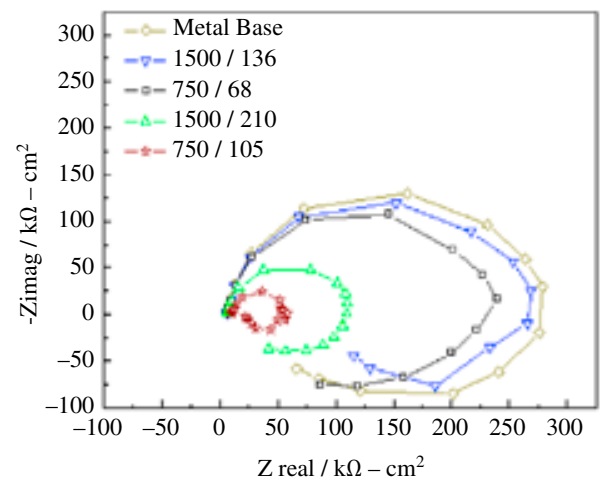

Figura 2. Diagramas de Nyquist de las muestras modificadas por PFA comparadas con el metal base.

En la Figura 3 se observa el circuito equivalente de aleación de magnesio AZ31-B (material base) y las soldaduras obtenidas por el proceso PFA, en donde $\mathrm{C}$ es la capacitancia de la doble capa y R1 la resistencia de transferencia de carga. Cuando están en paralelo la respuesta se caracteriza por la presencia de un semicírculo. El siguiente paso que permite obtener un símil de una reacción electroquímica y que complica el análisis es la resistencia en serie al circuito paralelo RC, que representa la resistencia de la solución (Rs). Esta situación tiene el efecto de transportar el semicírculo a valores mayores en el eje de la impedancia real (Z') del gráfico; la resistencia R2 y la inductancia L5 están asociadas a fenómenos de adsorción-desorción de especies en la superficie del electrodo, fenómenos que alteran el potencial del electrodo y la velocidad de corrosión del metal. Los circuitos equivalentes que han sido propuestos para modelar este tipo de espectros de impedancia pueden incluir elementos inductivos o combinaciones RC con valores negativos [16, 17].

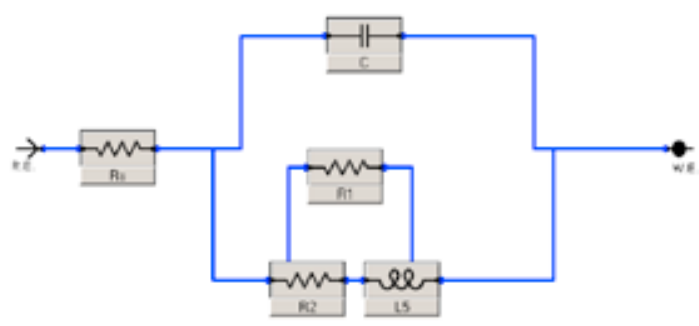

Figura 3. Circuito equivalente obtenido para los espectros de la Figura 2.

En la Figura 4 se muestran las curvas potenciodinámicas Tafel. En la obtenida para la aleación de magnesio AZ31B (metal base) se observa la menor densidad de corrosión en comparación con las soldaduras, lo cual muestra que después de la soldadura el material ha sufrido cambios en su estructura debido al proceso mecánico y térmico. Esto se ve reflejado en los valores que se exponen en la Tabla 3. Al compararlos, se aprecia que el proceso PFA con una relación de $\mathrm{Vr} / \mathrm{Va}$ de 1500/136 registra la mayor resistencia a la corrosión. El valor de densidad y velocidad de corrosión es similar al hallado para el material base. En una relación de $\mathrm{Vr} / \mathrm{Va}$ alta se observa que para $1500 / 136$ y $750 / 68(11,02)$ presentan los menores valores de velocidad de corrosión y para los de menor relación, cercanos a 7 , produce un aumento elevado en la densidad y la velocidad de corrosión debido al cambio microestructural que se generó con la relación $\mathrm{Vr} / \mathrm{Va}$.

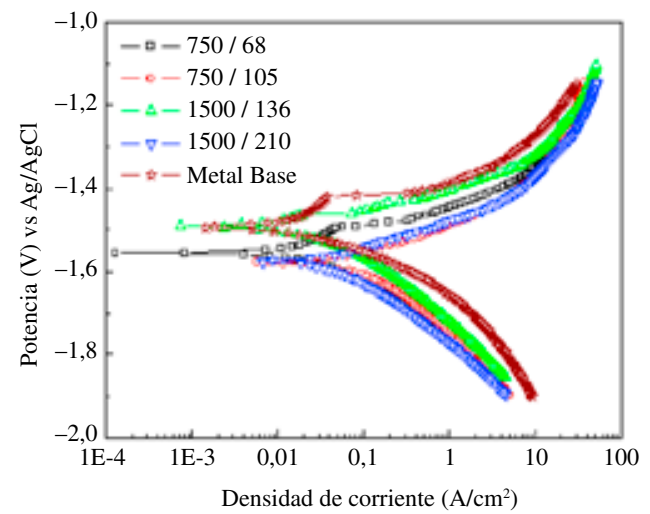

Figura 4. Curvas potenciodinámicas Tafel de la aleación de magnesio y las soldaduras obtenidas por el proceso PFA. 
$\mathrm{Al}$ calcular la densidad de corriente de intercambio, posterior al análisis de los datos obtenidos experimentalmente (Tabla 3), se puede determinar que el proceso está bajo control anódico. Esto indica una reacción más lenta en el paso del electrón a la superficie del metal y de su ión metálico a la superficie. Además, la película que se forma sobre la superficie del magnesio, un hidróxido, hace que este proceso sea más complejo. Dado esto, se puede afirmar que el proceso de soldadura modifica la superficie en mayor o menor grado dependiendo de las variables, lo cual se ve claramente reflejado en los resultados obtenidos. Adicionalmente, en el proceso cinético las corrientes de corrosión son muy susceptibles de sufrir cambios en la superficie, hecho que no se evidencia de igual manera con el potencial.

Tabla 3. Parámetros de las curvas potenciodinámicas Tafel de la aleación de magnesio y las soldaduras obtenidas por el proceso PFA.

\begin{tabular}{|c|c|c|c|}
\hline Muestra & $\begin{array}{c}\text { Ecorr }(\mathbf{m V} \\
\text { vs Ag/AgCI) }\end{array}$ & $\begin{array}{c}\text { Icorr } \\
\left(\boldsymbol{\mu A} / \mathbf{c m}^{2}\right)\end{array}$ & Vcorr (mpy) \\
\hline Metal base & -1490 & 8,2 & 4,4 \\
& $(3 \%)$ & $(0.23 \%)$ & $(0.10 \%)$ \\
\hline $1500 / 136$ & -1490 & 11,2 & 6,0 \\
$(11)$ & $(1 \%)$ & $(0.15 \%)$ & $(0.25 \%)$ \\
\hline $750 / 68$ & -1560 & 25,5 & 13,7 \\
$(11)$ & $(2 \%)$ & $(0.35 \%)$ & $(0.39 \%)$ \\
\hline $1500 / 210$ & -1570 & 52,2 & 28,0 \\
$(7)$ & $(3 \%)$ & $(0.42 \%)$ & $(0.45 \%)$ \\
\hline $750 / 105$ & -1580 & 93,2 & 50,0 \\
$(7)$ & $(4 \%)$ & $(0.38 \%)$ & $(0.36 \%)$ \\
\hline
\end{tabular}

\section{Análisis microestructural}

El cordón de soldadura por PFA está conformado por diferentes zonas, dependiendo del tipo y del grado de transformación sufrida durante el proceso. En la Figura 5 se observa la zona de transición entre el material base ubicado en el extremo inferior derecho de la imagen y la zona agitada en el extremo superior izquierdo. La microestructura del metal base está compuesta por grandes granos equiaxiales con tamaños variables entre 50 y $120 \mu \mathrm{m}$, acompañados de precipitados dispersos de fases intermetálicas del tipo AlxMny, mientras que la microestructura característica de la zona agitada está compuesta por granos finos equiaxiales y homogéneos de tamaño aproximado de $10 \mu \mathrm{m}$ con precipitados intermetálicos también finos. En la región intermedia se aprecia el cambio gradual del tamaño de los granos, además de unas bandas de deformación plástica resultantes del movimiento rotatorio del pin dentro del metal durante el proceso. Estas diferencias microestructurales forman una celda de corrosión que actúa como fuerza impulsora del proceso corrosivo.

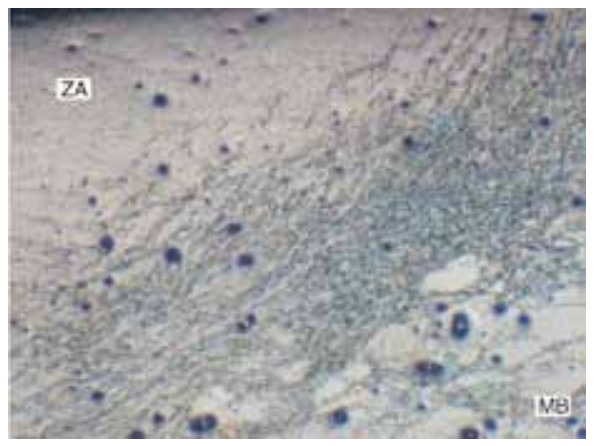

Figura 5. Microestructura del cordón de soldadura por PFA. ZA: Zona Agitada, MB: Metal base. 100X.

Las condiciones de soldadura determinan la cantidad de calor generado y sus efectos sobre la microestructura y las propiedades de la soldadura por PFA. Al aumentar la relación de velocidad de rotación/velocidad de soldadura crecen el calor generado en el cordón así como el tamaño de grano en la zona agitada.

En la Figura 6 se muestra una imagen de microscopia electrónica de barrido hecha sobre la superficie de las muestras, modificadas después de haber sido sometidas al proceso de evaluación electroquímica. Se puede observar una película que presenta grietas, no es compacta sino totalmente heterogénea. Esta capa superficial está compuesta por óxidos complejos de aluminio y magnesio e hidróxido de magnesio.

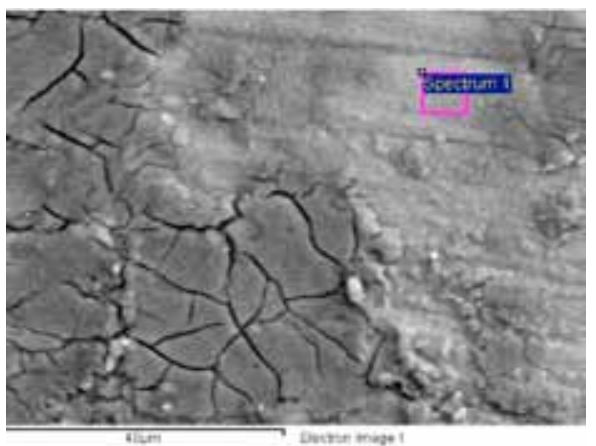

Figura 6. Micrografía SEM de la superficie de las muestras modificadas por PFA luego del proceso de corrosión. 500X. 
Adicionalmente, se observan sales formadas por cloro y sodio.

La disolución del magnesio se inicia con la adsorción de los iones hidroxilo sobre su superficie, y el hidróxido de magnesio es producido por la reacción electroquímica. Los iones de cloro también se hallan en la película; fácilmente penetran la película de hidróxido y forman una sal de cloruro básica.

$$
\begin{gathered}
\mathrm{Mg} \rightarrow \mathrm{Mg}^{2+}+2 \mathrm{e}^{-} \text {Reacción anódica } \\
2 \mathrm{H}_{2} \mathrm{O}+2 \mathrm{e}^{-} \rightarrow 2 \mathrm{OH}^{-}+\mathrm{H}_{2} \uparrow \text { Reacción catódica }
\end{gathered}
$$

Las reacciones de corrosión de aleaciones y de magnesio puro son similares en medios neutros y alcalinos. La reacción global puede expresarse como:

$$
\mathrm{Mg}+2 \mathrm{H}_{2} \mathrm{O}=\mathrm{Mg}(\mathrm{OH})_{2}+\mathrm{H}_{2} \uparrow \text { Reacción total }
$$

Los productos de corrosión formados sobre las superficies de aleaciones de magnesio de la serie AZ están formados por tres capas en las que existen variaciones en la concentración tanto de oxígeno como de magnesio y el contenido de aluminio y cinc se incrementa hacia el interior [18, 19].

Después de remover los productos de corrosión y observar las superficies de las muestras, se determinó que el tipo de corrosión es general. De acuerdo con algunas investigaciones, se sugiere que hay corrosión localizada que presenta picado. Sin embargo, esto está relacionado con el tiempo de inmersión de la prueba [18].

\section{CONCLUSIONES}

$\mathrm{Al}$ aumentar la relación de velocidad de rotación/ velocidad de avance en el proceso de fricciónagitación la velocidad de corrosión es menor, lo que indica que mejora la resistencia a la corrosión.

Se encontró que el tipo de corrosión producida es de carácter general y que los productos de corrosión corresponden a óxidos e hidróxidos de aluminio y magnesio.

\section{AGRADECIMIENTOS}

Esta investigación fue financiada por la Universidad Militar Nueva Granada Bogotá, Colombia.

\section{REFERENCIAS}

[1] F. Franco, H. Sánchez, D. Betancourt y O. Murillo. "Soldadura por fricción-agitación de aleaciones ligeras, una alternativa a nuestro alcance". Revista Latinoamericana de Metalurgia y Materiales. Vol. S1, No 3, pp. 1369-1375. Julio 2009.

[2] W.M. Thomas, E.D. Nicholas and S.D. Smith. "FSW-Tool developments". TMS Annual Meeting and exhibition $\mathrm{Al}$ joining. Vol. 1, Issue 1, pp. 1-12. January, 2001.

[3] A. Wendt, K. Weiss, A. Ben-Dov, M. Bamberger and B. Bronfin. "Magnesium Technology". TMS Warrendale. Vol. 15, Issue 2, pp. 269-273. February, 2005.

[4] S. Schumann and H. Friedrich. "Effect of Friction Stir Processing on Microstructure and Mechanical Properties of a Cast-Magnesium". Rare Earth Alloy. Mater. Sci. Forum. Vol. 51, Issue 3, pp. 419-422. April, 2003.

[5] Z.Y. Ma. "Friction Stir Processing Technology: A Review". Metall. Mater. Trans. Vol. 39A, Issue 3, pp. 642-658. March, 2008.

[6] C.I. Chang, C.J. Lee and J.C. Huang. "Relationship between grain size and Zener-Holloman parameter during friction stir processing in AZ31 Mg alloys". Scripta Mater. Vol. 51, Issue 6, pp. 509-514. July, 2004.

[7] B.M. Darras, M.K. Khraisheh, F.K. Abu-Farha and M.A. Omar. "Friction stir processing of commercial AZ31 magnesium alloy". Journal of Materials Processing Technology. Vol. 191, Issue 1-3, pp. 77-81. March, 2007.

[8] Z.Y. Ma, A.L. Pilchak, M.C. Juhas and J.C. Williams. "Microstructural refinement and property enhancement of cast light alloys via friction stir processing". Scripta Mater. Vol. 58, Issue 5, pp. 361-366. November, 2008.

[9] R.C. Zeng, J. Chen, W. Dietzel, R. Zettler, J.F. dos Santos, M.L. Nascimento and K.U. Kainer. "Corrosion of friction stir welded magnesium alloy AM50". Corrosion Science. Vol. 51, Issue 8, pp. 1738-1746. May, 2009.

[10] M. Kannan, W. Dietzel, R. Zeng, R. Zettler and J.F. dos Santos. "A study on the SCC susceptibility of friction stir welded AZ31 Mg sheet". Materials Science and Engineering A. 
Vol. 460-461, Issue 1, pp. 243-250. January, 2007.

[11] Z. Rong-chang. "Review of studies on corrosion of magnesium alloys". Transactions of Nonferrous Metals Society of China. Vol. 16, Supplement 2, pp. 763-771. June, 2006.

[12] C. Liu, D.L. Chen, S. Bhole, X. Cao and M. Jahazi. "Polishing-assisted galvanic corrosion in the dissimilar friction stir welded joint of AZ31 magnesium alloy to 2024 aluminum alloy". Materials Characterization. Vol. 60, Issue 5, pp. 370-376. May, 2009.

[13] C. Ying-liang and W. Hai-Ian. "Corrosion properties of AZ31 magnesium alloy and protective effects of chemical conversion layers and anodize coatings". Transactions of Nonferrous Metals Society of China. Vol. 17, Issue 3, pp. 502-508. May, 2007.

[14] ASTM G1-03. "Standard Practice for Preparing, Cleaning, and Evaluating Corrosion Test Specimens". West Conshohocken, PA, American Society for Testing and Materials. 2003.

[15] ASTM G3-89. "Standard Practice for Conventions Applicable to Electrochemical Measurements in Corrosion Testing”. West
Conshohocken, PA, American Society for Testing and Materials. 2010.

[16] J. Zhao, F. Jiang and H. Jian. "Comparative investigation of tungsten inert gas and friction stir welding characteristics of $\mathrm{Al}-\mathrm{Mg}-\mathrm{Sc}$ alloy plates". Materials and Design. Vol. 31, Issue 1, pp. 306-311. January, 2010.

[17] A. Squillace, A. De Fenzo and G. Giorleo. "A comparison between FSW and TIG welding techniques: modifications of microstructure and pitting corrosion resistance in AA 2024-T3 butt joints". Journal of Materials Processing Technology. Vol. 152, Issue 1, pp. 97-105. October, 2004.

[18] G. Ben Hamua, D. Eliezer and L. Wagner. "The relation between severe plastic deformation microstructure and corrosion behavior of AZ31 magnesium alloy". Journal of Alloys and Compounds. Vol. 468, Issue 1-2, pp. 222-229. January, 2009.

[19] N. Afrin, D.L. Chena and X. Cao. "Microstructure and tensile properties of friction stir welded AZ31B magnesium alloy". Materials Science and Engineering A. Vol. 472, Issue 1-2, pp. 179-186. January, 2008 . 